\title{
Anterior segment optical coherence tomography for imaging the anophthalmic socket
}

\author{
Mandeep S. Sagoo (iD ${ }^{1} \cdot$ Stephen Bell ${ }^{1,2} \cdot$ David Carpenter $^{1}{ }^{1} \cdot$ Gordon Bott $^{2} \cdot$ Naoko Hara $^{3} \cdot$ Udo Schmidt $^{4}$. \\ Marie Restori ${ }^{1}$
}

Received: 3 October 2019 / Accepted: 15 October 2019 / Published online: 28 October 2019

(c) The Royal College of Ophthalmologists 2019

\section{To the Editor:}

Non-invasive imaging by optical coherence tomography has revolutionised the management of ophthalmic patients, providing higher resolution of anatomical data to almost 'in vivo biopsy' resolution and the ability for 3D reconstruction [1]. Anterior segment OCT (AS-OCT) has been harnessed evaluating corneal disease, the iridocorneal angle in glaucoma [2], iridociliary [3] and conjunctival [4] tumours, the eyelid punctum [5] and the tear film, even in prosthetic eyes [6].

Currently, to manufacture an ocular prosthetic following enucleation an alginate impression mould is taken of the anophthalmic socket. Herein we report proof of concept that AS-OCT can be used to image the anterior orbit, potentially replacing the invasive technique of alginate impression moulding.

The CASIA2 AS-OCT (Tomey, Nagoya, Japan) has a fast scanning speed $(50,000 \mathrm{~A}$-scans/s) using a Fourier Domain Swept Source, enabling a $16 \mathrm{~mm}$ window composed of 800 A-scans and radial scan of 128 slices to a depth of $13 \mathrm{~mm}$ with an axial resolution of $10 \mu \mathrm{m}$, taking $2.4 \mathrm{~s}$. The eyelids can be retracted and the position of gaze altered to obtain optimal sections. An overall threedimensional view or two-dimensional sectioning in any radial plane is possible. The three-dimensional view can be rotated and a useful gonioscopic (or tangential) three-

Mandeep S. Sagoo

Mandeep.sagoo@nhs.net

1 National Institute for Health Research Biomedical Research Centre for Ophthalmology at Moorfields Eye Hospital NHS Foundation Trust and UCL Institute of Ophthalmology, London, UK

2 Ocupye Ltd, Warwick, UK

3 Tomey, Nagoya, Japan

4 Tomey GmbH, Nürnberg, Germany dimensional view is also available. A monochrome photograph of the eye is simultaneously collected.

A 70-year-old male with left raised intraocular pressure from iris ring melanoma (maximum elevation $1 \mathrm{~mm}$ ) underwent photography (Fig. 1a), anterior segment B-mode ultrasound examination and AS-OCT (Figs. 1b-d and 2a) diagnostic imaging. Enucleation was performed with insertion of a $22 \mathrm{~mm}$ vicryl mesh-wrapped hydroxyapatite implant. Histopathology of the enucleated eye confirmed ring melanoma.

After informed consent, repeat AS-OCT scans were taken of the anophthalmic socket (Fig. 2) in various directions of gaze, with and without a standard transparent polymethyl methacrylate (PMMA) conformer (Fig. 2b-h). The small aperture in the conformer clearly visible on the AS-OCT image acted as a landmark in central AS-OCT sections, with a monochrome photograph displaying the meridian of the AS-OCT scan. In the central section the effect of the PMMA conformer on overestimation of depth is noted as the underlying tissue appears to lie more posteriorly (Fig. 2f). This suggests that the system over estimates the separation of the tissue and conformer due to the refractive index of PMMA. An oblique nasal two-dimensional section through the standard conformer shows areas of touch, representing compression of tissue (Fig. 2g) and identify regions of potential discomfort in a prosthesis.

The alginate impression mould of the socket was used to make a transparent PMMA bespoke conformer with the same base curve properties as the eventual prosthesis. A central aperture was used as a landmark (Fig. 2h). This conformer was seen to lie considerably more proud to the conjunctiva than the standard conformer.

AS-OCT is used routinely in the assessment of anterior segment tumours, but in this report, we show that it can also be used to image the anophthalmic socket. This gives an idea of the curvature of the covered implant, but scans taken with a standard conformer in situ are useful in imaging the fit of a possible prosthesis. Using a custom-made conformer, the fit of the eventual prosthesis can be judged 
Fig. 1 Pre-enucleation images of the left eye with iris ring melanoma. a Colour photograph showing left eye with iris ring melanoma. b Three-dimensional gonioscopic view AS-OCT image through 4 o'clock meridian: raised lesion at pupillary edge. $\mathbf{c}$ Central normal horizontal two-dimensional ASOCT image through 3 o'clock d Horizontal two-dimensional AS-OCT image showing a reflective lesion (arrow) at pupillary edge (adjacent photo shows meridian of AS-OCT)

Fig. 2 Post-enucleation imaging of the left socket. a

Monochrome photograph from AS-OCT pre-enucleation left eye. b Monochrome photograph from AS-OCT post-enucleation of left socket. c AS-OCT in a vertical plane through anophthalmic socket shown in $\mathbf{b}$. d Tangential "gonioscopic" three-dimensional view of conjunctival surface in anophthalmic socket shown in $\mathbf{b}$. e Monochrome photograph from AS-OCT showing standard PMMA conformer with central circular aperture in situ post enucleation. $\mathbf{f}$ Two-dimensional AS-OCT image showing standard conformer and underlying socket: the aperture highlights effect of PMMA on measurement of separation of conformer posterior surface to conjunctiva (causes an overestimate in separation). g Oblique nasal twodimensional AS-OCT view (see adjacent photo for meridian) showing apposition of conformer to conjunctiva, probably represents tissue compression. h Twodimensional AS-OCT image showing a bespoke conformer with central aperture in situ: conformer posterior surface based on alginate impression (multiple reflection artefacts noted)
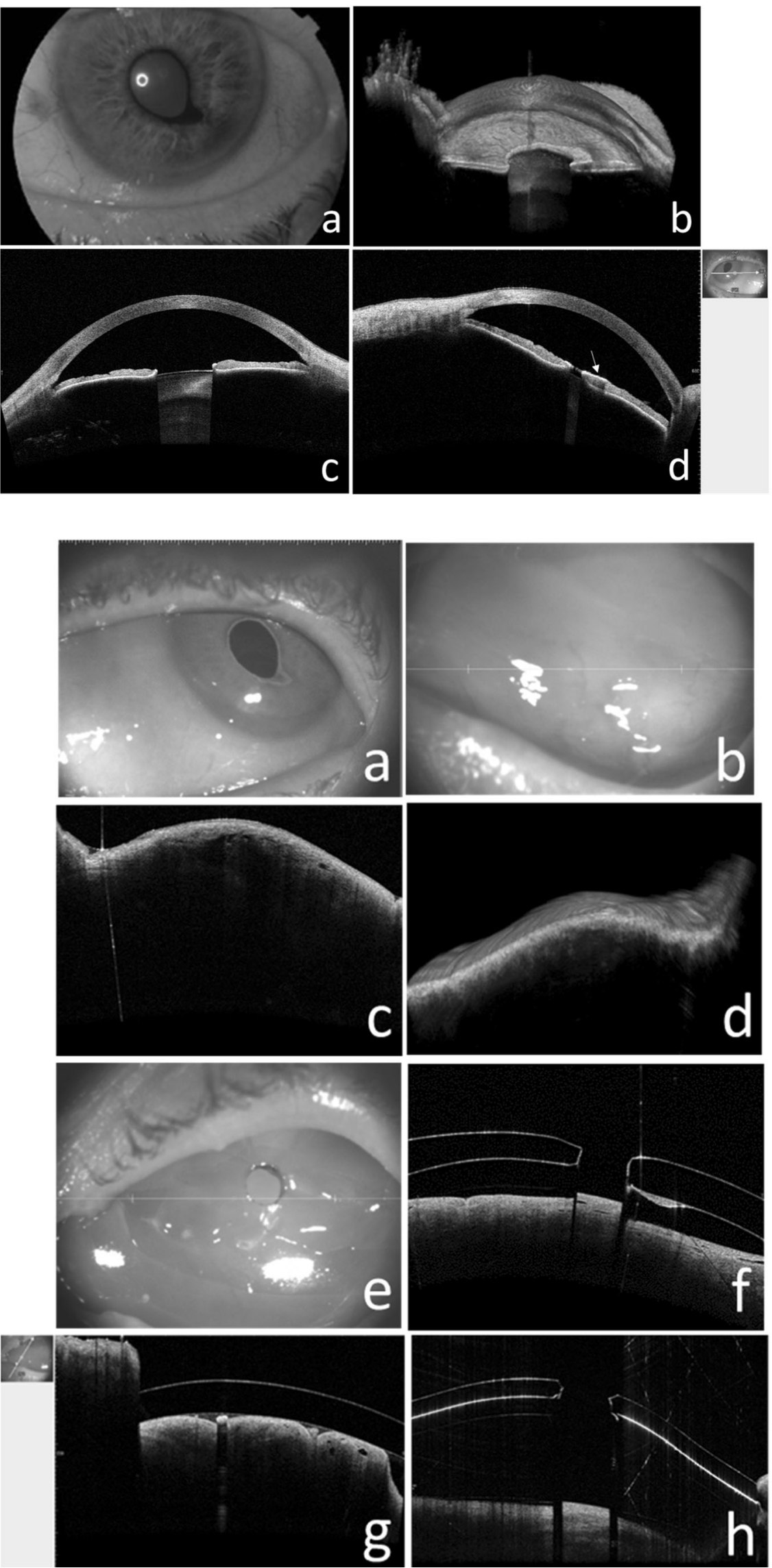
using the AS-OCT. We believe that AS-OCT will eventually replace alginate impression moulding required to manufacture a bespoke prosthesis. In this way a time-consuming, invasive and uncomfortable process can be replaced with a painless, non-invasive one that takes $2.4 \mathrm{~s}$.

Acknowledgements This report is independent research funded in part by the National Institute for Health Research under its Invention for Innovation (i4i) Programme (Grant reference number II-BP-081710009). The views expressed in this publication are those of the authors and not necessarily those of the NHS, the National Institute for Health Research or the Department of Health and Social Care.

\section{Compliance with ethical standards}

Conflict of interest The authors declare that they have no conflict of interest.

Publisher's note Springer Nature remains neutral with regard to jurisdictional claims in published maps and institutional affiliations.

\section{References}

1. Huang D, Swanson EA, Lin CP, Schuman JS, Stinson WG, Chang W, et al. Optical coherence tomography. Science. 1991;254: $1178-81$.

2. Ang M, Baskaran M, Werkmeister RM, Chua J, Schmidl D, Aranha Dos Santos V, et al. Anterior segment optical coherence tomography. Prog Retin Eye Res. 2018;66:132-56.

3. Hau SC, Papastefanou V, Shah S, Sagoo MS, Restori M, Cohen V. Evaluation of iris and iridociliary body lesions with anterior segment optical coherence tomography versus ultrasound B-scan. Br J Ophthalmol. 2015;99:81-6.

4. Venkateswaran N, Galor A, Wang J, Karp CL. Optical coherence tomography for ocular surface and corneal diseases: a review. Eye Vis. 2018;5:13.

5. Singh S, Ghosh A, Rath S. Imaging of proximal lacrimal system with time domain anterior segment optical coherence tomography in Asian Indian population. Orbit. 2017;36:251-5.

6. Kim SE, Yoon JS, Lee SY. Tear measurement in prosthetic eye users with fourier-domain optical coherence tomography. Am J Ophthalmol. 2010;149:602-7 e1. 